\title{
Prefrontal Cortical Kappa Opioid Receptors Attenuate Responses to Amygdala Inputs
}

\author{
Hugo A Tejeda ${ }^{1,5}$, Ashley N Hanks ${ }^{2}$, Liam Scott ${ }^{2}$, Carlos Mejias-Aponte ${ }^{3}$, Zoë A Hughes ${ }^{2}$ and \\ Patricio O'Donnell ${ }^{*, 1,2,4}$
}

'Department of Anatomy and Neurobiology, University of Maryland School of Medicine, Baltimore, MD, USA; ${ }^{2}$ Neuroscience and Pain Research Unit, Pfizer, Cambridge, MA, USA; ${ }^{3}$ Neuronal Networks Section, National Institute on Drug Abuse, Baltimore, MD, USA; ${ }^{4}$ Department of Psychiatry, University of Maryland School of Medicine, Baltimore, MD, USA

\begin{abstract}
Kappa opioid receptors (KORs) have been implicated in anxiety and stress, conditions that involve activation of projections from the basolateral amygdala (BLA) to the medial prefrontal cortex (mPFC). Although KORs have been studied in several brain regions, their role on mPFC physiology and on BLA projections to the mPFC remains unclear. Here, we explored whether KORs modify synaptic inputs from the BLA to the mPFC using in vivo electrophysiological recordings with electrical and optogenetic stimulation. Systemic administration of the KOR agonist U69,593 inhibited BLA-evoked synaptic responses in the mPFC without altering hippocampus-evoked responses. IntramPFC U69,593 inhibited electrical and optogenetic BLA-evoked synaptic responses, an effect blocked by the KOR antagonist nor-BNI. Bilateral intra-mPFC injection of the KOR antagonist nor-BNI increased center time in the open field test, suggesting an anxiolytic effect. The data demonstrate that mPFC KORs negatively regulate glutamatergic synaptic transmission in the BLA-mPFC pathway and anxiety-like behavior. These findings provide a framework whereby KOR signaling during stress and anxiety can regulate the flow of emotional state information from the BLA to the mPFC.

Neuropsychopharmacology (20I5) 40, 2856-2864; do:I 0.1038/npp.20I5.I38; published online 3 June 2015
\end{abstract}

\section{INTRODUCTION}

Dynorphin acting on kappa opioid receptors (KORs) is a powerful mediator of behavioral stress reactivity and negative effect (Bruchas et al, 2010; Tejeda et al, 2012; Van't Veer and Carlezon, 2013b). KORs are present in limbic and cortical regions involved in these functions, such as the medial prefrontal cortex (mPFC) and basolateral amygdala (BLA), and mPFC KORs may have a crucial role in anxiety and stress. Indeed, mPFC KOR signaling is necessary and sufficient for KOR-mediated aversion (Bals-Kubik et al, 1993; Tejeda et al, 2013). Furthermore, KOR antagonism in the BLA produces anxiolytic effects (Bruchas et al, 2009; Knoll et al, 2011). Thus, KORs may mediate negative affective states by modulating neuronal activity in the MPFC and BLA. As BLA projections to the mPFC are critical for stress and anxiety, it is possible that these projections mediate behavioral effects of KORs.

The neural mechanisms responsible for behavioral effects of KOR activation are only beginning to be understood. KOR immunoreactivity is presynaptically located in excitatory

*Correspondence: Professor P O'Donnell, Neuroscience and Pain Research Unit, Pfizer, 610 Main Street, Cambridge, MA 02139, USA, Tel: + | 67| 395 0838, Fax: + | 845474 4276,

E-mail: patricio.odonnell@pfizer.com

${ }^{5}$ Current address: National Institute of Drug Abuse, 25I Bayview Boulevard, Room 05A31 I, Baltimore, MD 21224, USA.

Received I 6 January 20 I4; revised 30 April 20 I5; accepted I May 20I5; accepted article preview online 14 May 2015 synapses in the rat mPFC (Svingos and Colago, 2002), and KOR activation decreases mPFC glutamate synaptic transmission via a presynaptic site of action (Tejeda et al, 2013). KORs also inhibit $\mathrm{K}^{+}$-evoked glutamate release from synaptosomes (Sbrenna et al, 1999). These observations suggest that $\mathrm{mPFC}$ KORs may shape glutamatergic synaptic transmission by inhibiting presynaptic glutamate terminals, but the source of the inputs being modulated is not known. As anatomical studies indicate the $\mathrm{MPFC}$ receives glutamatergic inputs from the BLA (Bacon et al, 1996; McDonald, 1996) and the BLA has a rich density of KOR receptor mRNA and immunoreactivity (DePaoli et al, 1994; Meng et al, 1993; Van't Veer et al, 2013a), it is conceivable that mPFC KORs are expressed in BLA terminals, thereby modulating glutamatergic inputs from this limbic structure. Here, we utilized in vivo electrophysiological techniques combined with optogenetics to determine whether KORs negatively regulate glutamatergic inputs from the BLA to the $\mathrm{MPFC}$.

\section{MATERIALS AND METHODS}

\section{Subjects}

Adult male Long-Evans rats (300-400 g, Charles River) and adult male C57BL/6J mice (22-25 g; Jackson Labs) were housed in temperature- and humidity-controlled facilities under a 12-h light/dark cycle with ad libitum chow and water. Experiments were conducted in accordance with the USPH Guide for the Care and Use of Laboratory Animals, 
and approved by the Institutional Animal Care and Use Committees of the University of Maryland School of Medicine and Pfizer.

\section{In vivo Extracellular Electrophysiology}

A subset of rats were treated with saline or nor-BNI $(10 \mathrm{mg} / \mathrm{kg}$, s.c.) $24 \mathrm{~h}$ prior to recording procedures to antagonize KORs. nor-BNI, similar to other KOR antagonists, produces long-lasting antagonism of KORs (Carroll and Carlezon, 2013). Rats were anesthetized with choral hydrate $(400 \mathrm{mg} / \mathrm{kg}$, i.p.) and fixed on a stereotaxic apparatus (Kopf Instruments). Anesthesia was maintained throughout the remainder of the experiment using continuous choral hydrate $(24-30 \mathrm{mg} / \mathrm{kg} / \mathrm{h})$ via an intraperitoneal catheter. Body temperature was maintained at $\sim 37^{\circ} \mathrm{C}$ using a probecontrolled heat pad (Fine Science Tools). Concentric bipolar stimulating electrodes $(0.5 \mathrm{~mm}$ diameter, $0.5 \mathrm{~mm}$ pole separation; Rhodes Medical Instruments) were lowered into the left BLA (3.0 mm caudal to bregma (AP); $4.9-5.0 \mathrm{~mm}$ lateral to midline (ML); $7.4 \mathrm{~mm}$ from dura (DV)) and in some cases also into the left fornix (AP: $-3.0 \mathrm{~mm}$; ML: 3.9$4.0 \mathrm{~mm}$; DV: -3.1 to $3.2 \mathrm{~mm}$ ). Coordinates were based on the rat brain atlas of Paxinos and Watson (1998).

Extracellular recording microelectrodes were pulled from 1.5-mm-diameter borosilicate glass (World Precision Instruments) using a vertical puller (Stoelting). Tips were broken back to obtain electrodes with 5-15 M 2 resistance recorded in situ. We utilized custom-built double-barrel glass pipettes for local drug pressure ejection as previously described (Dilgen et al, 2013). Pressure ejection pipettes were pulled from 1.0-mm-diameter borosilicate glass $(0.25 \mathrm{~mm}$ ID; A-M Systems). A paper rule was taped to the drug barrel to measure the amount of drug delivery; one millimeter was delivered for $\sim 50 \mathrm{nl}$ of solution. Drug pipettes were filled with artificial cerebrospinal fluid (aCSF) containing the AMPA and NMDA receptor antagonists CNQX $(50 \mu \mathrm{M})$ and AP-5 (100 $\mu \mathrm{M})$, respectively, the GABA-A receptor antagonist picrotoxin $(1 \mathrm{mM})$, or the KOR agonist U69,593 $(1 \mathrm{mM})$, and $<0.01 \%$ Chicago Sky Blue. Extracellular recording microelectrodes were filled with $0.5 \mathrm{M}$ sodium chloride. Microelectrodes were lowered into the cortex using a hydraulic manipulator (Trent Wells) and electrode resistance was determined. Signals were passed through a headstage to a Neurodata Intracellular Amplifier (Cygnus), subsequently amplified, and continuously monitored on a digital oscilloscope (Fluke), a multimeter (Tektronix), and an audio monitor (Grass). Signals were digitized at $20 \mathrm{kHz}$ using a Digidata (Molecular Devices), and acquired with Axoscope 9 software (Molecular Devices) for offline analysis. Local field potentials were lowpass filtered at $100 \mathrm{~Hz}$.

Microelectrodes were lowered through the MPFC while the BLA or fornix was stimulated $(0.1-0.5 \mathrm{~ms}$ duration; 0.4$1.0 \mathrm{~mA} ; 0.2 \mathrm{~Hz}$ ) until a consistent monosynaptic-evoked field excitatory postsynaptic potential (fEPSP) was encountered. Antidromic activation of mPFC-projecting BLA neurons produces responses with a mean latency of $12-21 \mathrm{~ms}$ (Likhtik et al, 2005; Rosenkranz and Grace, 1999). In our study, a response was deemed monosynaptic if the onset latency was $<10 \mathrm{~ms}$ and had a negative-going peak $\leqslant 25 \mathrm{~ms}$ from stimulation. Moreover, synaptic responses demonstrated monotonic increases in fEPSP slope with increasing electrical stimulation intensity in line with monosynaptic responses. Furthermore, we measured the slope of the fEPSP, as we have previously demonstrated that early components of evoked synaptic potentials are composed of excitatory responses, using in vivo intracellular recordings (Dilgen et al, 2013). In experiments assessing the effects of systemic U69,593 on BLA- and fornix-evoked responses, the BLA or fornix were stimulated every $30 \mathrm{~s}$ with a 15 -s delay between BLA and fornix. Following a 30-min baseline recording, the KOR agonist U69,593 $(0.32 \mathrm{mg} / \mathrm{kg}$; s.c.) was systemically injected and recordings were continued. Mean responses were calculated for every 15-min interval. For pressure ejection experiments, baseline was assessed for $5 \mathrm{~min}$ while the BLA was electrically stimulated every $5 \mathrm{~s}$. Mean responses were calculated for every 30-s interval. aCSF, CNQX/AP-5, picrotoxin, or U69,593 was pressure ejected using a Toohey Pressure System IIe (Toohey Systems; 40 psi; 5-10 ms duration) $500 \mathrm{~ms}$ before BLA stimulation over a 5 -min period. This resulted in delivery of $\sim 50 \mathrm{nl}$ of aCSF or drug. A maximum of two sites were sampled from each rat in local drug experiments. At least $45 \mathrm{~min}$ were allowed after drug delivery before advancing the micropipette $>750 \mu \mathrm{m}$ ventrally to search for another recording site.

\section{Optogenetics}

Rats were anesthetized with $1-2 \%$ isoflurane. An adenoassociated virus (serotype 5) expressing channelrhodopsin-2YFP (ChR2-YFP) under the control of the CaMKII promoter was bilaterally microinjected into the BLA (AP: $-3.0 \mathrm{~mm}$; ML: $\pm 5.0 \mathrm{~mm}$; DV: $7.4 \mathrm{~mm}$ from dura; $0.5 \mu \mathrm{l}$ ). Electrophysiological experiments were conducted 7-9 weeks after viral infection. These experiments were conducted as described above with the exception that an optical fiber $(100 \mu \mathrm{m})$ coupled to a laser diode (473 nm emission; Thor Labs) was fixed to the drug pipette $(350-400 \mu \mathrm{m}$ above the recording electrode tip) to optically activate ChR2-expressing BLA terminals in the $\mathrm{mPFC}$ and eject drug near the recording site. Chicago Sky Blue was not included in aCSF or drug pipettes to circumvent any effects of the dye on light spread. Optical stimulation consisted of a pulse of $473 \mathrm{~nm}$ light $(8-15 \mathrm{~mW}$; $1 \mathrm{~ms}$ duration) delivered every $5 \mathrm{~s}$.

\section{Drugs}

U69,593 and nor-BNI were supplied by the Research Technology Branch of NIDA (Rockville, MD), and norBNI used for behavioral testing was synthesized by Pfizer Worldwide Medicinal Chemistry. CNQX, AP-5, and picrotoxin were purchased from Sigma-Aldrich. U69,593 was dissolved in $0.1 \mathrm{~N} \mathrm{HCl}$, and subsequently brought to a $\mathrm{pH}$ of 7.4 with $\mathrm{NaOH}$. CNQX, AP-5, picrotoxin, and nor-BNI were diluted in aCSF for local drug administration. Nor-BNI was dissolved in saline for systemic administration.

\section{Behavioral Testing}

All mice were singly housed in Innocage individually vented cage racks for at least 7 days prior to testing. Mice were randomly assigned to 1 of 5 treatment groups (systemic: vehicle or nor-BNI $(10 \mathrm{mg} / \mathrm{kg})$; intra-mPFC vehicle or nor-BNI (2 ng or $2 \mu \mathrm{g}$ bilaterally)). For intra-mPFC 
a
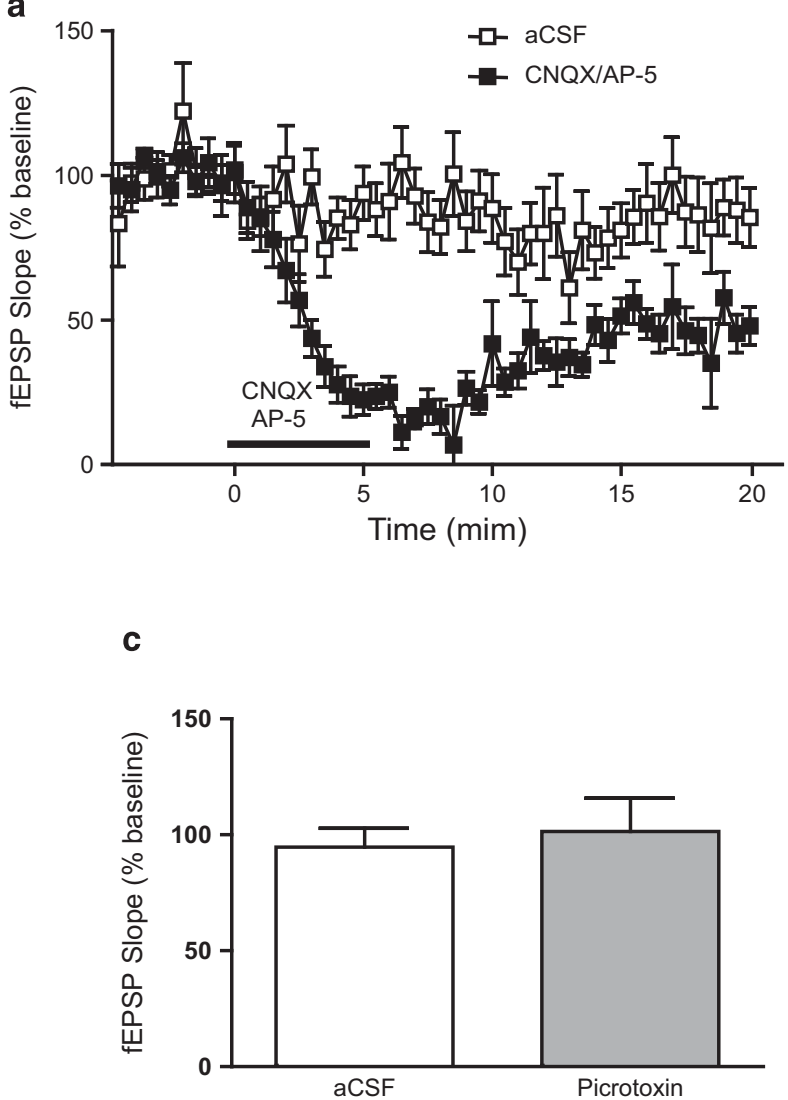

b
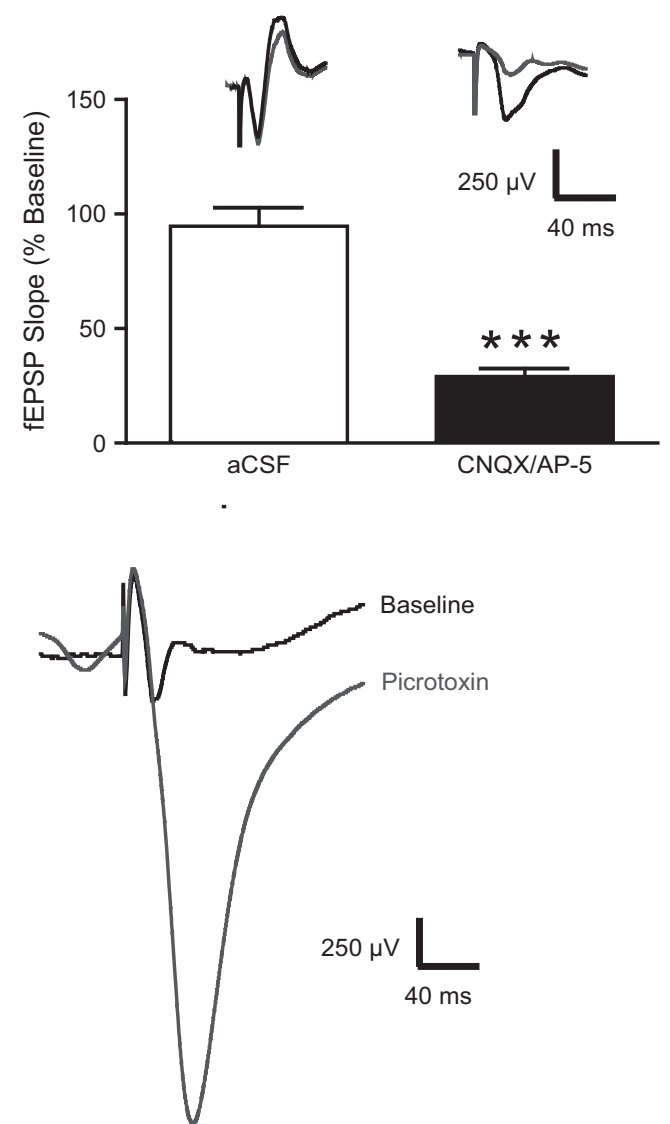

Figure I BLA-evoked synaptic responses in the mPFC are blocked by pressure-ejected glutamate receptor antagonists. (a) Time course of the effects of pressure-ejected aCSF (white squares; $n=11$ sites in seven rats) or CNQX/AP-5 (black squares; $n=7$ sites in five rats) on the BLA-evoked fEPSP slope. Horizontal bar depicts the period of CNQXIAP-5 pressure ejection. (b) Mean fEPSP slope expressed as a percentage of baseline in the 5-min window after the last puff of aCSF or CNQXIAP-5. (****) reflects a significant difference from aCSF controls. Representative traces of BLA-evoked fEPSPs at baseline (black trace) and after aCSF or CNQX/AP-5 (gray trace) are shown above their corresponding bars. (c) Mean fEPSP slope expressed as a percentage of baseline in the window following picrotoxin pressure ejection. Right, representative traces of BLA-evoked fEPSPs at baseline and (black trace) and after picrotoxin (gray trace). Note that picrotoxin modifies later components of the fEPSP without affecting the slope. Scale bars: $0.5 \mathrm{mV}$ and $20 \mathrm{~ms}$. In this and subsequent figures, data points depict mean \pm SEM. aCSF, artificial cerebrospinal fluid; BLA, basolateral amygdala; fEPSP, field excitatory postsynaptic potential; mPFC, medial prefrontal cortex.

administration, mice were anesthetized using $1-2 \%$ isoflurane and placed into a stereotaxic frame. Mice were given injections of saline and carprofen $(5 \mathrm{mg} / \mathrm{kg}$, s.c.) for hydration and analgesia. Two holes were drilled into the skull and nor-BNI or vehicle was injected bilaterally into the mPFC (AP: $+1.7 \mathrm{~mm}$; ML: $\pm 0.3 \mathrm{~mm}$; DV: $-2.5 \mathrm{~mm}$, from bregma) over 1 min using a Hamilton syringe attached to a stereotaxic adapter syringe $(0.1 \mu \mathrm{l}$ per site). The syringe was left in position for $2 \mathrm{~min}$ post delivery to allow for diffusion. The incision was closed using medical glue. To assess effects of systemic administration, separate mice were dosed with vehicle or nor-BNI (10 mg/kg, s.c.). After 6 days, mice were brought into the locomotor testing room and allowed to acclimate for $1 \mathrm{~h}$ prior to being placed into the Animal Activity Chambers $(40 \times 40 \times 40 \mathrm{~cm}$; Accuscan Instruments $)$ housed in sound-attenuating chambers (lighting 250-400 lux) with normal background noise. Time spent in the center, total distance traveled $(\mathrm{cm})$, and vertical activity were recorded using infrared beams for a period of $60 \mathrm{~min}$. Treatment was assigned randomly, but ensuring that all treatments were evenly distributed over time to avoid any effect of time of day. The surgeon was blinded to the drug being administered, as the assistant surgeon loaded the stereotaxic adapter syringe. A separate experimenter tested and analyzed the behavior of both the infused mice and systemic administered mice using automated Versamax Animal Activity Chambers. After data analysis was complete, the experimenter was unblinded to the groups. No outliers were removed from the assay. Two mice administered norBNI (2 ng) did not survive surgery and thus were not tested; this accounts for the uneven sample sizes.

\section{Histology}

Upon termination of the experiments, rats were euthanized and brains were removed. For optogenetic experiments, rats were transcardially perfused with saline followed by $4 \%$ paraformaldehyde. Brains were postfixed overnight in $4 \%$ paraformaldehyde, rinsed in PBS, and switched to $30 \%$ sucrose with $1 \%$ azide. Sections $(40-50 \mu \mathrm{m})$ were obtained using a freezing microtome. During sectioning, the location of Chicago Sky Blue as an index of the recording site was 
verified. Sections were Nissl-stained, cover-slipped, and examined under a microscope to verify stimulating electrode placements and recording microelectrode track placement. Fluorescent DAPI counterstain was utilized for optogenetic studies.

\section{Data Analyses}

Time-course experiments for systemic administration of U69,593 were analyzed using a two-way ANOVA with time as a within-subjects factor and stimulation site as a betweensubjects factor. Time-course experiments for pressure ejection experiments were analyzed using a two-way ANOVA with time as a within-subjects factor and drug as a between-subjects factor. Mean change from baseline values were analyzed using the Student's $t$-test or one-way ANOVA. Behavioral data were analyzed using GraphPad Prism software (Version 5.02; GraphPad Software, La Jolla, CA). The effects of treatment on time spent in center, total distance traveled, and vertical activity within the Versamax apparatus were analyzed using one-way ANOVA followed by Dunnett's $t$-tests for post hoc comparisons.

\section{RESULTS}

\section{Systemic KOR Activation Inhibits BLA, but not Hippocampal Projections to the PFC}

To assess modulation of inputs to the MPFC by KOR, we first determined whether BLA-evoked fEPSP responses were glutamatergic. The onset latency of BLA-evoked responses was less than $10 \mathrm{~ms}$, consistent with a monosynaptic response, and local administration of a mixture of the AMPA antagonist CNQX and the NMDA antagonist AP-5 significantly decreased fEPSP slope relative to aCSF (Figure $1 \mathrm{a}$ and $\mathrm{b})$. Repeated measures ANOVA revealed a main effect of time $\left(\mathrm{F}_{(49,686)}=8.08 ; p<0.0001\right)$, a main effect of drug $\left(\mathrm{F}_{(1686)}=24.95 ; p=0.0002\right)$, and a time $\times$ drug interaction $\left(\mathrm{F}_{(49,686)}=4.54 ; p<0.0001\right)$. A comparison of the mean fEPSP slope (expressed as a percentage of baseline) in the 5-min bin (10-15 min) after pressure ejection between aCSF and CNQX/AP-5 groups revealed a significant difference $\left(t_{(16)}=6.117 ; p<0.0001\right)$. These results indicate the slope of BLA-evoked fEPSP is dependent on glutamatergic transmission.

We have previously shown that BLA inputs to the $\mathrm{MPFC}$ provide strong feedforward inhibition into the $\mathrm{mPFC}$ by activating fast-spiking interneurons (Dilgen et al, 2013). It is therefore possible that BLA-evoked feedforward inhibition contributes to the fEPSP slope. We tested for this possibility by examining the effects of pressure ejection of the GABA-A channel blocker picrotoxin near our recording site. Although fEPSP amplitude was increased in some cases, pressure ejection of picrotoxin failed to modify the slope of the BLAevoked fEPSP (Figure $2 c ; t_{(18)}=0.42 ; p=0.67$ ). Thus, BLAevoked fEPSP responses provide a reliable measure of glutamatergic transmission in the BLA-mPFC pathway.

We next determined the effects of systemic U69,593 on glutamatergic transmission in the BLA-mPFC and hippocampus-mPFC pathways. As KOR mRNA expression is largely absent in the output regions of the hippocampal formation (DePaoli et al, 1994; Meng et al, 1993; Van't Veer a

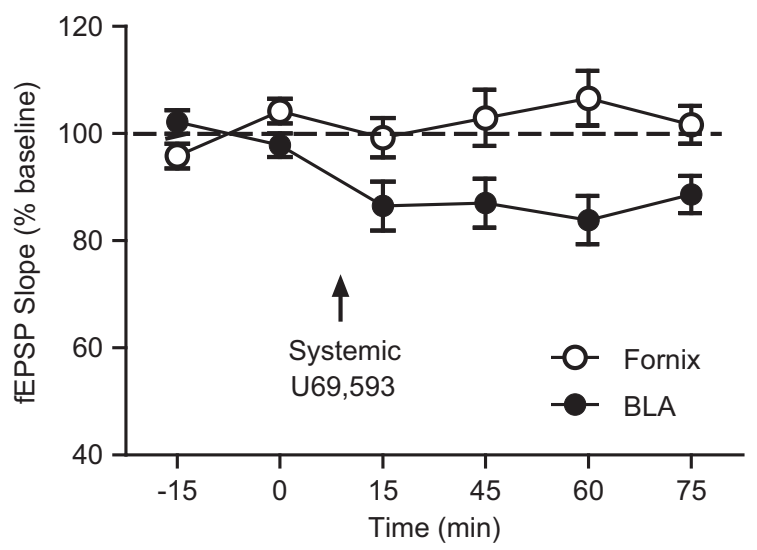

b
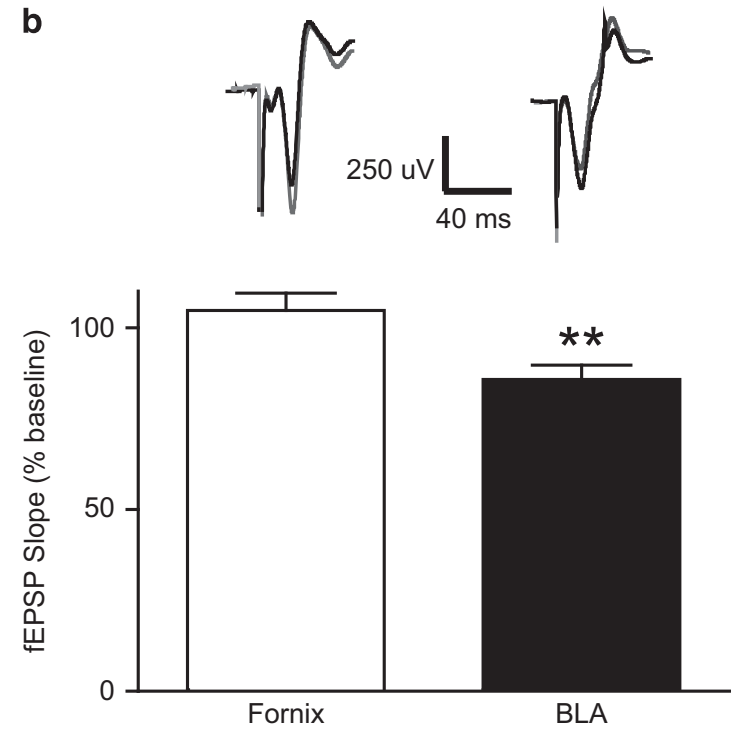

Figure 2 Systemic administration of a KOR agonist inhibits glutamatergic fEPSPs in a pathway-specific manner. (a) Time course of the effects of systemic $U 69,593$ on fornix-evoked (white circles; $n=1 \mid$ rats) and BLAevoked (black circles; $n=10$ rats) fEPSP slope. (b) Mean fEPSP slope expressed as a percentage of baseline in the 30-min window after systemic U69,593 injection. (**) reflects a significant difference from fornix-evoked synaptic responses. Representative traces of fornix- and BLA-evoked fEPSPs at baseline (black trace) and after systemic U69,593 administration (gray trace) are shown above their corresponding group. Scale bars: $0.5 \mathrm{mV}$ and $20 \mathrm{~ms}$. BLA, basolateral amygdale; fEPSP, field excitatory postsynaptic potential; KOR, kappa opioid receptor.

et al, 2013a), we did not expect this pathway to be modulated by KORs. Systemic U69,593 administration reduced the slope of fEPSPs evoked by BLA stimulation, but not stimulation of the fornix, the fiber bundle containing ventral hippocampal axons that innervate the mPFC (Figure 2). Repeated measures ANOVA revealed no effect of time $\left(\mathrm{F}_{(5,95)}=1.76 ; p=0.1286\right)$, a main effect of the stimulation site $\left(\mathrm{F}_{(1,95)}=9.58 ; p=0.006\right)$, and a time $\times$ stimulation site interaction $\left(\mathrm{F}_{(5,95)}=4.74 ; p=0.0007\right)$. Post hoc analysis of baseline fEPSP slopes of BLA and fornix responses 1545 min after systemic U69,593 administration revealed that BLA synaptic responses were inhibited relative to fornix responses $\left(t_{(19)}=3.06 ; p<0.0064\right)$. These results suggest that KORs inhibit glutamatergic synaptic transmission arriving in the $\mathrm{mPFC}$ from the BLA, but not hippocampus. 


\section{mPFC KORs Inhibit the BLA-PFC Pathway}

The effects of systemic KOR activation reported above could be due to receptors located in a variety of structures. To determine whether KORs located in the mPFC modulate glutamatergic transmission in the BLA-mPFC pathway, we assessed the effects of intra-mPFC U69,593 pressure ejection on BLA-evoked fEPSPs in rats that had received systemic administration of saline or the KOR antagonist nor-BNI $\sim 24 \mathrm{~h}$ prior to recordings. U69,593 pressure ejection within the $\mathrm{mPFC}$ reduced BLA-evoked fEPSP slope, and this effect was blocked by nor-BNI (Figure 3a and b). Repeated measures ANOVA revealed a main effect of time $\left(\mathrm{F}_{(49,1421)}=3.31\right.$; $p<0.0001)$, no main effect of drug $\left(\mathrm{F}_{(2,1421)}=1.13 ; p=0.34\right)$, and a significant time $\times$ drug interaction $\left(\mathrm{F}_{(98,1421)}=1.33\right.$; $p=0.02)$. One-way ANOVA of the mean percent of baseline for the 5-min period following U69,593 ejection showed a significant effect $\left(\mathrm{F}_{(2,35)}=5.191 ; p=0.011\right)$. Post hoc analysis showed that U69,593 inhibited BLA-evoked fEPSP slope relative to aCSF $(p<0.05)$. Pretreatment with nor-BNI blocked the ability of pressure-ejected U69,593 to inhibit BLA-evoked synaptic responses in the $\operatorname{mPFC}(p<0.05)$, suggesting that U69,593-mediated inhibition of BLA-evoked fEPSPs is due to action at the KOR. These results suggest that KORs in the mPFC negatively modulate BLA inputs.

The inhibitory effects of mPFC KOR activation may be different depending on stimulation intensity. The slope of BLA-evoked fEPSPs was higher with increased stimulation intensities (Figure 3c), with maximal responses at $1.0 \mathrm{~mA}$. $\mathrm{U} 69,593$ produced a downward shift in the $\mathrm{MPFC}$ response curve BLA stimulation. Repeated measures ANOVA revealed a main effect of stimulation intensity $\left(\mathrm{F}_{(4,36)}=102.54\right.$; $p<0.0001)$, a main effect of drug $\left(\mathrm{F}_{(1,36)}=13.33 ; p=0.005\right)$, and a stimulation intensity $\times$ drug interaction $\left(\mathrm{F}_{(4,36)}=6.98\right.$; $p<0.0001)$. Post hoc tests revealed a significant difference at $0.6-1.0 \mathrm{~mA}$ intensities $(p<0.05)$, but not at lower current intensities. The findings suggest that increasing current intensity does not overcome intra-mPFC U69,593-mediated inhibition of the BLA-mPFC pathway, but rather reveals inhibitory influence of KOR on this pathway.

\section{mPFC KORs Inhibit Optogenetic Activation of the BLA-mPFC Pathway}

BLA electrical stimulation could result in activation of fibers of passage and/or antidromic activation of $\mathrm{mPFC}$ efferents in addition to activation of BLA projection neurons. To verify U69,593 was specifically acting on BLA-mPFC projections, we repeated experiments using optogenetics instead of electrical BLA stimulation. Intra-BLA AAV-ChR2 injection resulted in robust expression of YFP in the BLA and in fibers in layers II/III and deep layers of the mPFC (Figure 4a). We first verified that optical activation of ChR2-expressing BLA fibers in the $\mathrm{mPFC}$ produced monosynaptic glutamatergic responses. Optical stimulation near the recording site evoked fEPSPs with a shorter onset latency and time to peak $(7.8 \pm 0.3 \mathrm{~ms})$ than fEPSPs evoked by BLA electrical stimulation $(18.6 \pm 1.1 \mathrm{~ms})$. We verified that optically evoked responses were glutamatergic, as intra-mPFC CNQX/AP-5 pressure ejection reduced the slope of optically evoked fEPSPs (Figure $4 \mathrm{~b}$ and $\mathrm{c}$ ). Repeated measures ANOVA revealed a main effect of time $\left(\mathrm{F}_{(49,539)}=12.35 ; p<0.0001\right)$, a main effect of drug $\left(\mathrm{F}_{(1539)}=22.77 ; p=0.0006\right)$, and a significant time $\times$ drug interaction $\left(\mathrm{F}_{(49,539)}=6.28 ; p<0.0001\right)$. A comparison of the fEPSP slope (expressed as a percentage of baseline) in the 5-min bin after pressure ejection between aCSF and CNQX/AP-5 groups revealed a significant difference $\left(t_{(11)}=6.659 ; p<0.0001\right)$. Thus, optical stimulation of ChR2-expressing BLA terminals evokes monosynaptic fEPSPs dependent on fast glutamatergic signaling.

Intra-mPFC U69,593 pressure ejection reduced the slope of optically evoked BLA fEPSPs in the mPFC (Figure 4d and e). Repeated measures ANOVA revealed a main effect of time $\left(\mathrm{F}_{(49,735)}=9.83 ; p<0.0001\right)$, a main effect of drug $\left(\mathrm{F}_{(1735)}=12.76 ; p=0.0028\right)$, and a significant time $\times$ drug interaction $\left(\mathrm{F}_{(49,735)}=3.11 ; p<0.0001\right)$. A comparison of the fEPSP slope (expressed as a percentage of baseline) in the 5 min after pressure ejection revealed a significant difference between aCSF and U69,593 groups $\left(t_{(15)}=5.099 ; p<0.0001\right)$. These results suggest that $\mathrm{mPFC}$ KORs selectively inhibit BLA afferents to the mPFC.

\section{Anxiolytic Effect of mPFC KOR Blockade}

The effects of intra-mPFC infusion of nor-BNI on locomotor activity were evaluated 6 days after treatment. The lower dose of nor-BNI ( $2 \mathrm{ng}$ ) caused a significant increase in time spent in the center of the Versamax apparatus $\left(\mathrm{F}_{(2,31)}=8.97\right.$; $p=0.0008)$ compared with vehicle $(q=0.63 \quad p<0.01$; Figure 5a). Total distance traveled $\left(\mathrm{F}_{(2,31)}=1.21 ; p=0.3098\right)$ and vertical activity $\left(\mathrm{F}_{(2,31)}=1.12 ; p=0.3391\right)$ were not significantly affected by treatment with nor-BNI ( $2 \mathrm{ng}$; Figure $5 \mathrm{~b}$ and $\mathrm{c})$. The higher dose of nor-BNI $(2 \mu \mathrm{g})$ had no effect on any of the measures (Figure $5 \mathrm{a}-\mathrm{c}$ ). There was a trend for center time to be higher in mice treated with vehicle systemically $v s$ intra-mPFC $(p=0.08$; Figure $5 \mathrm{a})$. Systemic administration of nor-BNI (10 mg/kg, s.c.) did not affect any behavioral measures (Figure $5 \mathrm{a}-\mathrm{c}$ ). The data indicate that mPFC KORs are sufficient to mediate anxiety-related behaviors.

\section{DISCUSSION}

Systemically administered U69,593 inhibited BLA-evoked synaptic responses, assessed with the slope of fEPSPs. This measure reflects evoked glutamate release, as it was also inhibited by locally applied AMPA and NMDA receptor antagonists. Systemic U69,593 was ineffective on hippocampus-evoked fEPSPs, suggesting that KOR regulation of glutamatergic limbic afferents to the mPFC is pathway specific. Local pressure ejection of U69,593 inhibited BLAevoked glutamatergic fEPSPs in the MPFC, and this effect was blocked by the KOR antagonist nor-BNI. Local U69,593 pressure ejection also inhibited optically evoked BLA synaptic responses in the $\mathrm{mPFC}$, suggesting that U69,593 action is specific to BLA terminals. Collectively, these results indicate that KORs in the MPFC negatively regulate limbic glutamatergic inputs in a pathway-specific manner.

Although there is evidence of KORs localized in the mPFC, their cellular distribution is not clear. KOR immunoreactivity is present in asymmetric synapses indicative of excitatory synapses (Svingos and Colago, 2002), but as the PFC receives glutamatergic inputs from diverse structures, it is not clear 
which terminals are modulated by KORs. Our data show that KORs gate BLA inputs to the mPFC. On the other hand, we failed to find an effect of systemic KOR activation on hippocampal inputs to the mPFC. This difference is consistent with high levels of KOR mRNA expression in the BLA, but not the output regions of the ventral hippocampus: the ventral subiculum or CA1 region (George et al, 1994; Mansour et al, 1994; Meng et al, 1993). A lack of homogenous regulation of all glutamatergic synapses explains our previous results where U69,593 produced a slight but significant decrease in the frequency of mini EPSPs in mPFC slices (Tejeda et al, 2013), as mini EPSPs are spontaneous events likely arising from both KOR-sensitive and KOR-insensitive terminals. It is also possible that KOR
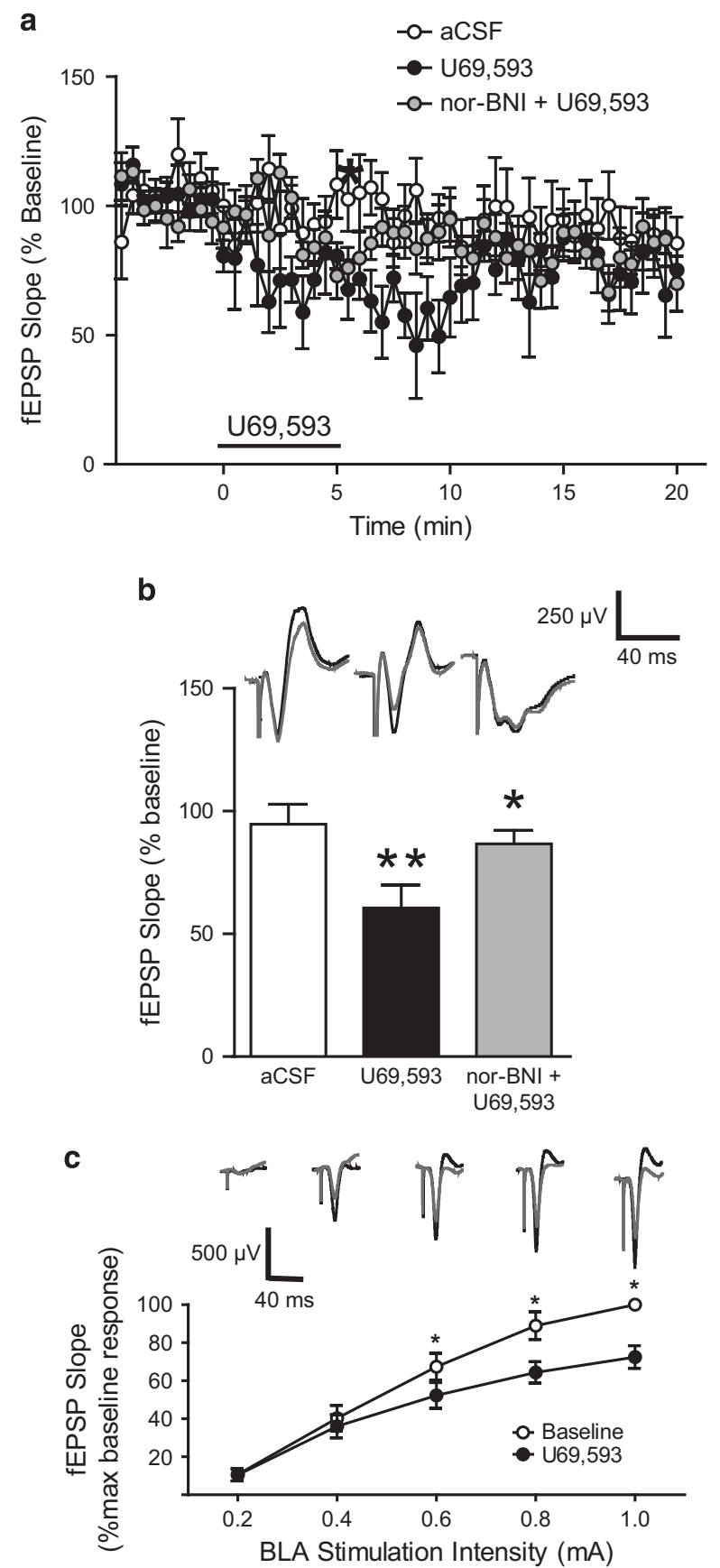

modulates other glutamatergic projections to the mPFC we did not explore here, such as the thalamus. A proportion of dopamine (DA) neurons co-express the glutamate transporter (Yamaguchi et al, 2011) and have been shown to co-release glutamate in the ventral striatum (Stuber et al, 2010; Tecuapetla et al, 2010). Electrical and chemical activation of the VTA produces fast glutamatergic synaptic responses in the mPFC (Lavin et al, 2005), suggesting that mesocortical DA neurons may also co-release glutamate. As KORs directly inhibit mPFC DA varicosities (Tejeda et al, 2013), KORs may also be inhibiting glutamate provided by VTA fibers. Inputspecific regulation of glutamatergic mPFC afferents by local KORs would provide a means to negatively regulate specific pathways in response to conditions that elevate mPFC dynorphin/KOR signaling.

KOR activation was more effective at inhibiting synaptic responses with increasing stimulation intensity. This enhanced effect could be due to additional recruitment of BLA fibers or a higher recruitment of local mPFC networks, which may unveil activation of a KOR-sensitive cell population. Optical stimulation of BLA terminals provided a means to determine KOR regulation of BLA to $\mathrm{MPFC}$ projections while avoiding activation of fibers of passage. Consistent with the notion that BLA electrical stimulation recruits nonspecific afferents to the MPFC not modulated by KOR, the inhibitory effects of U69,593 on BLA-evoked optical fEPSPs were less variable compared with effects on BLA electrically evoked fEPSPs, though the magnitude of inhibition was similar. However, as ChR2-eYFP was expressed under the control of the CaMKII promoter, it is also possible that neurons actively expressing CaMKII in the BLA are more strongly modulated by KORs, and these neurons are differentially recruited with increasing stimulation intensity.

KOR modulation of BLA inputs to the $\mathrm{MPFC}$ provides a neural basis, whereby dynorphin and KOR can affect emotional processing and anxiety. Communication between the amygdala and frontal cortex is in fact implicated in emotional processing, stress, and anxiety (Kim et al, 2011; Townsend and Altshuler, 2012). Many conditions can increase dynorphin in the mPFC, eventually affecting BLA

\footnotetext{
Figure $3 \mathrm{mPFC}$ KORs negatively modulate glutamatergic synaptic transmission in the BLA-mPFC pathway. (a) Time course of the effects of pressure-ejected artificial cerebrospinal fluid (aCSF) (white circles; $n=1$ I sites in seven rats), $U 69,593$ (black circles; $n=13$ sites in seven rats), or $\cup 69,593$ in nor-BNI pre-treated rats (gray circles; $n=12$ sites in seven rats) on the BLAevoked fEPSP slope. (b) Mean fEPSP slope expressed as a percentage of baseline in the 5-min window after the last puff of aCSF or U69,593 in rats pretreated with saline or nor-BNI $\sim 24 \mathrm{~h}$ prior to electrophysiological recordings. (**) reflects a significant difference from aCSF controls. (*) reflects a significant difference between saline- and nor-BNI-pretreated rats that were challenged with U69,593 pressure ejection. Representative traces of BLAevoked fEPSPs at baseline (black trace) and after aCSF or U69,593 (gray trace) are shown above their corresponding bars. Scale bars: $0.5 \mathrm{mV}$ and $20 \mathrm{~ms}$. (c) Mean BLA-evoked fEPSP normalized to maximal response during baseline at different stimulation intensities ( $n=10$ sites in 10 rats). (*) reflects a significant difference from baseline post U69,593 pressure ejection. Scale bars: $0.5 \mathrm{mV}$ and $20 \mathrm{~ms}$. Representative traces of BLA-evoked fEPSPs in the mPFC with increasing BLA stimulation intensity $(0.2-1.0 \mathrm{~mA}$ ) at baseline (black trace) or after U69,593 administration (gray trace) are shown above the corresponding points. BLA, basolateral amygdale; fEPSP, field excitatory postsynaptic potential; KOR, kappa opioid receptor, mPFC, medial prefrontal cortex.
} 


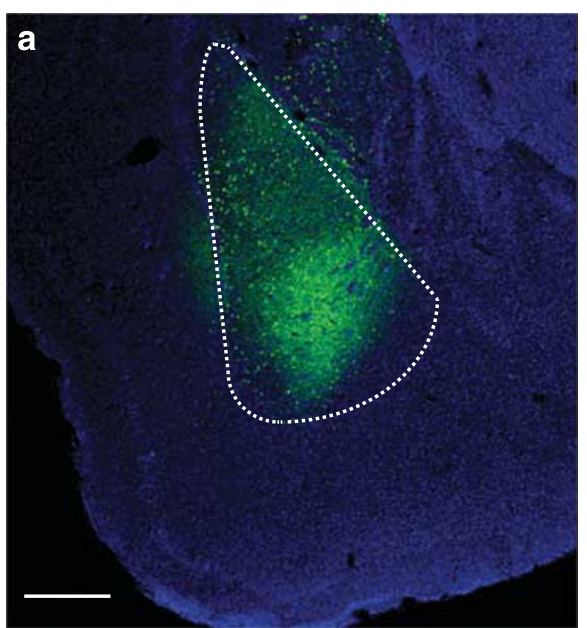

C

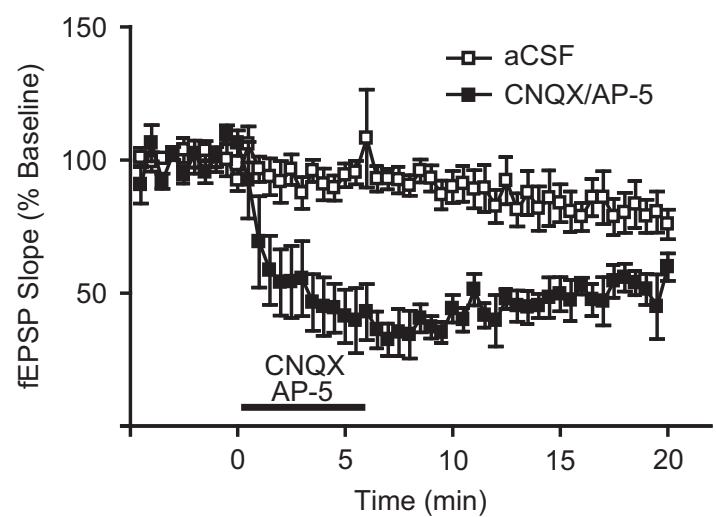

e

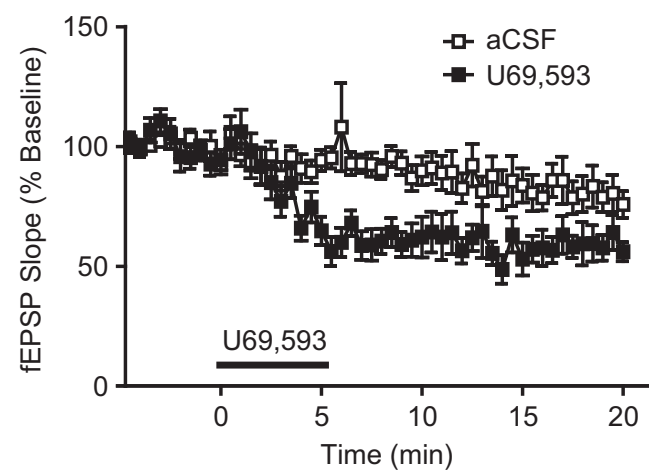

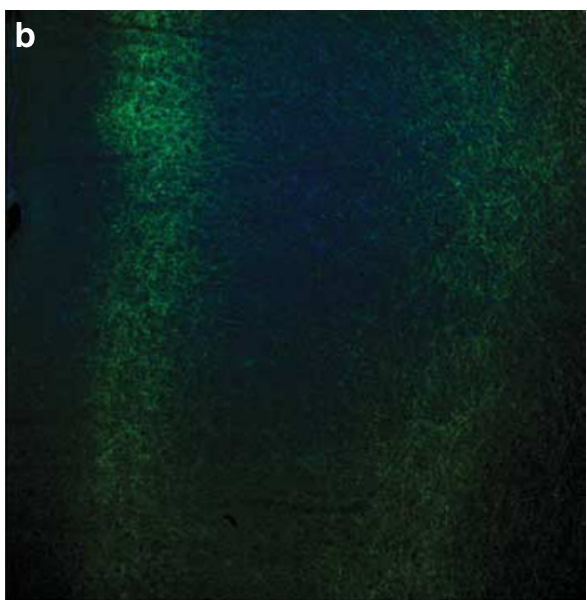

d
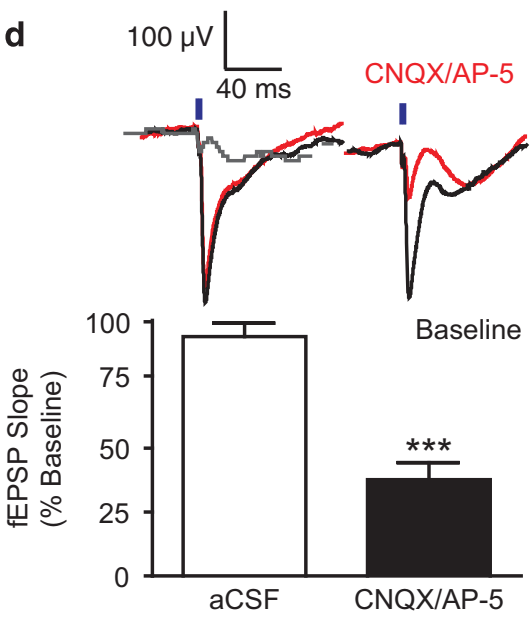

f

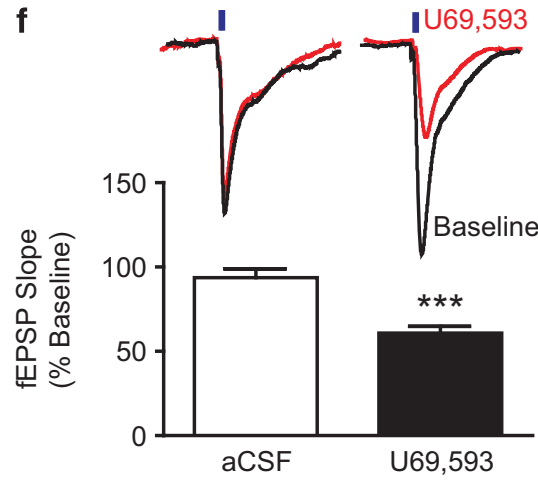

Figure 4 Optically evoked synaptic responses are blocked by CNQX/AP-5 pressure ejection. (a) Photomicrograph of ChR2-YFP expression (green) and DAPI-stained nuclei (blue) in the BLA. The dotted line depicts the boundaries of the BLA. Inset scale bar: $500 \mu \mathrm{m}$. (b) ChR2-YFP expression (green) in the mPFC. (c) Time course of the effects of pressure-ejected aCSF (white squares; $n=8$ sites in four rats) or CNQX/AP- 5 (black squares; $n=5$ sites in three rats) on the slope of BLA optically evoked fEPSP. (d) Top, representative traces of BLA-evoked fEPSPs at baseline (black traces) and after aCSF or CNQXIAP-5 (red traces). In gray is a representative trace of a site where blue-light pulses did not evoke a significant fEPSP response. Vertical blue bars depict I-ms blue-light pulses. Bottom, mean fEPSP slope expressed as a percentage of baseline in the 5-min window after the last puff of aCSF or CNQX/AP-5. (****) reflects a significant difference from aCSF controls. Black bar depicts the period of CNQX/AP-5 pressure ejection. (e) Time course of the effects of pressure ejected aCSF (white circles; $n=8$ sites in four rats) or U69,593 (black circles; $n=9$ sites in six rats) on the BLA optically evoked fEPSP slope. (f) Mean fEPSP slope expressed as a percentage of baseline in the 5-min window after the last puff of aCSF or U69,593. (****) reflects a significant difference from aCSF controls. Black bar depicts the period of U69,593 pressure ejection. Representative traces of BLA-evoked fEPSPs at baseline (black traces) and after aCSF or U69,593 (gray traces) are shown above their corresponding group. Blue lines depict I-ms blue-light pulses. Scale is the same as in d. aCSF, artificial cerebrospinal fluid; BLA, basolateral amygdale; ChR2-YFP, channelmodopsin-2-YFP; DAPI, 4',6-diamidino-2-phenylindole; fEPSP, field excitatory postsynaptic potential; mPFC, medial prefrontal cortex. 

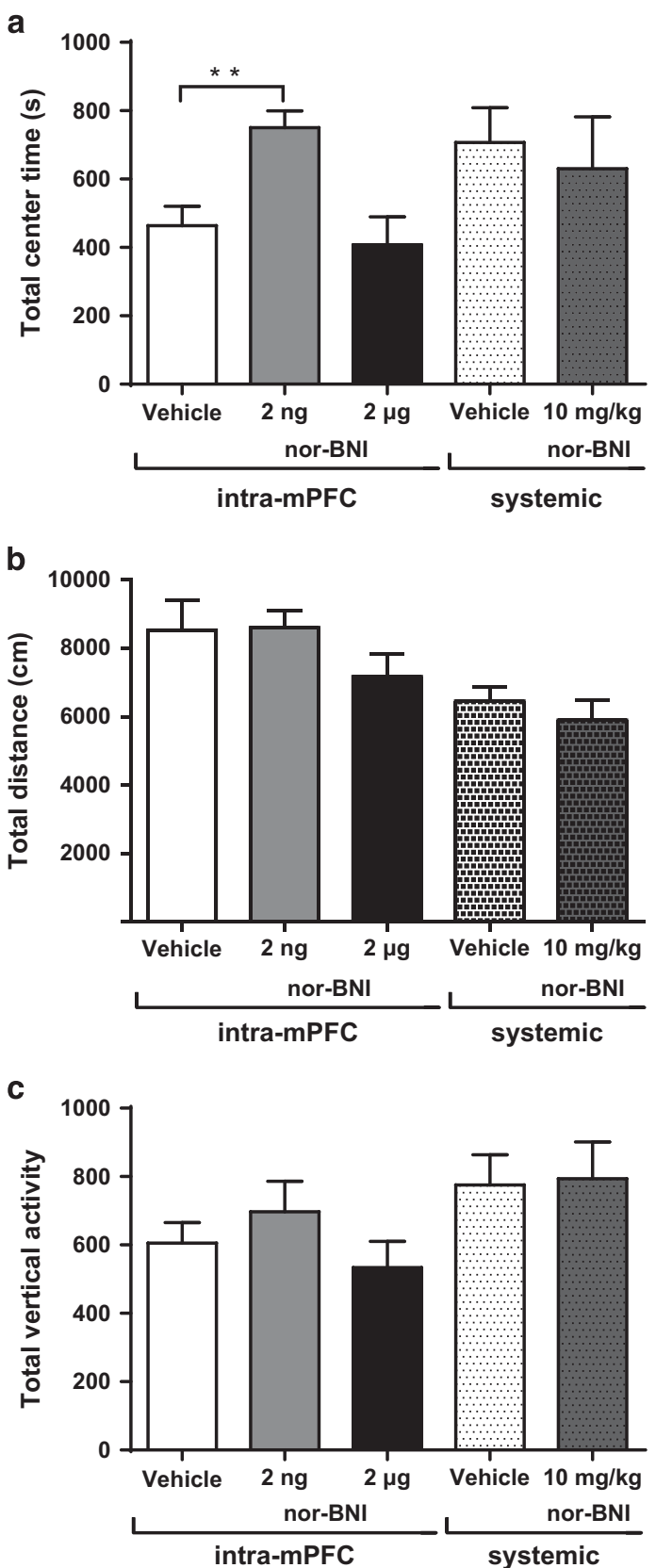

Figure 5 Effects of intra-mPFC and systemic administration of nor-BNI on locomotor activity. (a) The lower dose of intra-mPFC nor-BNI (2 ng) significantly increased center time, whereas the higher dose $(2 \mu \mathrm{g})$ as well as the systemic dose $(10 \mathrm{mg} / \mathrm{kg}$ ) had no effect. (b) Nor-BNI (2 ng, $2 \mu \mathrm{g}$, or $10 \mathrm{mg} / \mathrm{kg}$ ) had no effect on total distance traveled. (c) Vertical activity was also not affected by nor-BNI $(2 \mathrm{ng}, 2 \mu \mathrm{g}$, or $10 \mathrm{mg} / \mathrm{kg})$. Bars represent the mean $(n=10-12) \pm S E M * * *<0.01$ vs vehicle. mPFC, medial prefrontal cortex.

inputs. There is post-mortem evidence of dynorphin and KOR upregulation in the PFC of alcoholics relative to controls (Bazov et al, 2013) or in subjects with a lifetime of psychostimulant use (Peckys and Hurd, 2001). Increased KOR signaling in PFC regions of alcoholics and psychostimulant users would be expected to result in a hypodopaminergic cortical state, as mPFC KORs inhibit cortical DA output (Tejeda et al, 2013) and alterations in glutamate synaptic transmission from the amygdala. mPFC KOR upregulation may contribute to deficits in PFC-dependent decision-making and cognition in alcohol and drug addiction. Furthermore, KORs increase impulsive behavior (Walker and Kissler, 2013), which is inhibited by mPFC activation. As mPFC KOR signaling is sufficient to produce aversion and necessary for KOR-mediated aversion (BalsKubik et al, 1993; Tejeda et al, 2013), it is possible that heightened PFC KOR signaling contributes to a negative affective state during abstinence. This is of importance as negative affective states are powerful mediators of negative reinforcement processes that drive addiction. Our data indicate that mPFC dynorphin and KORs can modulate afferent responses dependent on BLA inputs. Indeed, intramPFC blockade of KOR elicited an increase in the time spent in the center of an activity box, suggesting KOR activation may be involved in anxiety-related behaviors and KOR blockade could have an anxiolytic profile. Interestingly, this effect was not observed at the high intra-mPFC dose of norBNI $(2 \mu \mathrm{g})$, which results in local concentrations well in excess of the $\mathrm{Ki}$ of nor-BNI for KOR $(0.8 \mathrm{nM})$ or with systemic nor-BNI administration. Whether this reflects an inverted U-shaped concentration response curve or a greater diffusion of nor-BNI within the brain was beyond the scope of these experiments. The time spent in the center was lower for mice dosed with vehicle intra-mPFC compared with the systemic route, indicating a lower level of anxiety in the systemically dosed groups. This also explains why systemic administration of nor-BNI did not have an anxiolytic-like effect. It is plausible that the surgical procedure increases the level of anxiety and increased tone at the KOR, which reveals an anxiolytic-like effect of intra-mPFC nor-BNI. Therefore, KORs may prove to be therapeutic targets for conditions with high affective components and compromised PFC function, such as alcohol and drug addiction, as well as anxiety and depression.

\section{FUNDING AND DISCLOSURE}

PO'D, ANH, LS and ZAH are employees and stockholders at Pfizer. The remaining authors declare no conflict of interest.

\section{ACKNOWLEDGMENTS}

We thank Dr Karl Deisseroth for the generous donation of the AAV-CamKII-ChR2 construct for the optogenetics part of the study. The study was supported by NIMH Grant R01 MH057683 (PO'D), a National Science Foundation Graduate Research Fellowship (HAT) and the Meyerhoff Graduate Fellowship (HAT).

\section{REFERENCES}

Bacon SJ, Headlam AJ, Gabbott PL, Smith AD (1996). Amygdala input to medial prefrontal cortex $(\mathrm{mPFC})$ in the rat: a light and electron microscope study. Brain Res 720: 211-219.

Bals-Kubik R, Ableitner A, Herz A, Shippenberg TS (1993). Neuroanatomical sites mediating the motivational effects of opioids as mapped by the conditioned place preference paradigm in rats. J Pharmacol Exp Ther 264: 489-495.

Bazov I, Kononenko O, Watanabe H, Kuntic V, Sarkisyan D, Taqi MM et al (2013). The endogenous opioid system in human 
alcoholics: molecular adaptations in brain areas involved in cognitive control of addiction. Addict Biol 18: 161-169.

Bruchas MR, Land BB, Chavkin C (2010). The dynorphin/kappa opioid system as a modulator of stress-induced and pro-addictive behaviors. Brain Res 1314: 44-55.

Bruchas MR, Land BB, Lemos JC, Chavkin C (2009). CRF1-R activation of the dynorphin/kappa opioid system in the mouse basolateral amygdala mediates anxiety-like behavior. PLoS One 4: e8528.

Carroll FI, Carlezon WA Jr (2013). Development of kappa opioid receptor antagonists. J Med Chem 56: 2178-2195.

DePaoli AM, Hurley KM, Yasada K, Reisine T, Bell G (1994). Distribution of kappa opioid receptor mRNA in adult mouse brain: an in situ hybridization histochemistry study. Mol Cell Neurosci 5: 327-335.

Dilgen J, Tejeda HA, O'Donnell P (2013). Amygdala inputs drive feedforward inhibition in the medial prefrontal cortex. J Neurophysiol 110: 221-229.

George SR, Zastawny RL, Briones-Urbina R, Cheng R, Nguyen T, Heiber $\mathrm{M}$ et al (1994). Distinct distributions of mu, delta and kappa opioid receptor mRNA in rat brain. Biochem Biophys Res Commun 205: 1438-1444.

Kim MJ, Gee DG, Loucks RA, Davis FC, Whalen PJ (2011). Anxiety dissociates dorsal and ventral medial prefrontal cortex functional connectivity with the amygdala at rest. Cereb Cortex 21: $1667-1673$

Knoll AT, Muschamp JW, Sillivan SE, Ferguson D, Dietz DM, Meloni EG et al (2011). Kappa opioid receptor signaling in the basolateral amygdala regulates conditioned fear and anxiety in rats. Biol Psychiatry 70: 425-433.

Lavin A, Nogueira L, Lapish CC, Wightman RM, Phillips PE, Seamans JK (2005). Mesocortical dopamine neurons operate in distinct temporal domains using multimodal signaling. J Neurosci 25: 5013-5023.

Likhtik E, Pelletier JG, Paz R, Pare D (2005). Prefrontal control of the amygdala. J Neurosci 25: 7429-7437.

Mansour A, Fox CA, Meng F, Akil H, Watson SJ (1994). Kappa 1 receptor mRNA distribution in the rat CNS: comparison to kappa receptor binding and prodynorphin mRNA. Mol Cell Neurosci 5: 124-144.

McDonald AJ (1996). Glutamate and aspartate immunoreactive neurons of the rat basolateral amygdala: colocalization of excitatory amino acids and projections to the limbic circuit. J Comp Neurol 365: 367-379.

Meng F, Xie GX, Thompson RC, Mansour A, Goldstein A, Watson SJ et al (1993). Cloning and pharmacological characterization of a rat kappa opioid receptor. Proc Natl Acad Sci USA 90: 9954-9958.
Paxinos G, Watson C (1998). The Rat Brainin Stereotaxic Coordinates4th edn.Academic Press.

Peckys D, Hurd YL (2001). Prodynorphin and kappa opioid receptor mRNA expression in the cingulate and prefrontal cortices of subjects diagnosed with schizophrenia or affective disorders. Brain Res Bull 55: 619-624.

Rosenkranz JA, Grace AA (1999). Modulation of basolateral amygdala neuronal firing and afferent drive by dopamine receptor activation in vivo. J Neurosci 19: 11027-11039.

Sbrenna S, Marti M, Morari M, Calo G, Guerrini R, Beani L et al (1999). L-glutamate and gamma-aminobutyric acid efflux from rat cerebrocortical synaptosomes: modulation by kappa- and mu- but not delta- and opioid receptor like-1 receptors. J Pharmacol Exp Ther 291: 1365-1371.

Stuber GD, Hnasko TS, Britt JP, Edwards RH, Bonci A (2010). Dopaminergic terminals in the nucleus accumbens but not the dorsal striatum corelease glutamate. J Neurosci 30: 8229-8233.

Svingos AL, Colago EE (2002). Kappa-Opioid and NMDA glutamate receptors are differentially targeted within rat medial prefrontal cortex. Brain Res 946: 262-271.

Tecuapetla F, Patel JC, Xenias H, English D, Tadros I, Shah F et al (2010). Glutamatergic signaling by mesolimbic dopamine neurons in the nucleus accumbens. J Neurosci 30: 7105-7110.

Tejeda HA, Counotte DS, Oh E, Ramamoorthy S, Schultz-Kuszak KN, Backman CM et al (2013). Prefrontal cortical Kappa-Opioid receptor modulation of local neurotransmission and conditioned place aversion. Neuropsychopharmacology 38: 1770-1779.

Tejeda HA, Shippenberg TS, Henriksson R (2012). The dynorphin/ kappa-opioid receptor system and its role in psychiatric disorders. Cell Mol Life Sci 69: 857-896.

Townsend J, Altshuler LL (2012). Emotion processing and regulation in bipolar disorder: a review. Bipolar Disord 14: 326-339.

Van't Veer A, Bechtholt AJ, Onvani S, Potter D, Wang Y, Liu-Chen LY et al (2013a). Ablation of Kappa-Opioid receptors from brain dopamine neurons has anxiolytic-like effects and enhances cocaineinduced plasticity. Neuropsychopharmacology 38: 1585-1597.

Van't Veer A, Carlezon WA Jr (2013b). Role of kappa-opioid receptors in stress and anxiety-related behavior. Psychopharmacology (Berl) 229: 435-452.

Walker BM, Kissler JL (2013). Dissociable effects of kappa-opioid receptor activation on impulsive phenotypes in wistar rats. Neuropsychopharmacology 38: 2278-2285.

Yamaguchi T, Wang HL, Li X, Ng TH, Morales M (2011). Mesocorticolimbic glutamatergic pathway. J Neurosci 31 8476-8490. 